\title{
Abordagem Laparoscópica do Câncer de Endométrio
}

\author{
Laparascopic Approach to Endometrial Cancer
}

Caio Parente Barbosa, Thomas Moscovitz Nelson Valente Martins, Ângela Mara Bentes de Souza

Ivania T. Soubhia, Ana Paula Santos Aldrighi

\begin{abstract}
RESUMO
Objetivo: apresentar uma nova abordagem laparoscópica para tratamento do câncer de endométrio.

Métodos: de fevereiro de 1996 à fevereiro de 1998, doze pacientes com câncer de endométrio, diagnosticadas por histeroscopia e biópsia, foram submetidas a linfadenectomia pélvica associada à histerectomia e salpingooforectomia por laparoscopia. A média de idade das pacientes foi de 58, 1 anos; o número médio de gestações foi 2,3 e o indice de massa corpórea médio foi de 28,6.

Resultados: o tempo anestésico médio foi de 4,8 horas. O tempo médio de internação foi de 3,3 dias. O número total de linfonodos retirados foi de 176, sendo 104 (59,1\%) do lado direito e 72 (40,9\%) do lado esquerdo. Não foram encontrados linfonodos comprometidos. $O$ número médio de linfonodos retirados por paciente foi de 18,5. Foram observadas duas complicações: em um caso houve necessidade de abandonar o procedimento por aumento da pressão endotraqueal e uma das pacientes evoluiu com formação de granuloma em cúpula vaginal.

Conclusões: nesta avaliação preliminar dos resultados de histerectomia total com anexectomia bilateral e lifadenectomia laparoscópica para o tratamento do câncer de endométrio, obtivemos resultados satisfatórios quanto ao estadiamento da neoplasia ao número de linfonodos retirados e quanto às complicações cirúrgicas observadas.
\end{abstract}

PALAVRAS-CHAVE: Histerectomia. Linfadenectomia. Cirurgia: complicações. Endométrio: adenocarcinoma. Laparoscopia.

\section{Introdução}

O câncer de endométrio é a doença maligna mais comum do trato genital feminino nos Estados Unidos da América ${ }^{5}$. No Brasil, segundo dados do

Serviço de Ginecologia do Hospital de Ensino da Faculdade de Medicina do ABC

Correspondência:

Caio Parente Barbosa

Al. Jauaperi, $n^{\circ} 732$ - Moema

04523-013 - São Paulo - SP
Ministério da Saúde entre 1981 e 1985, ocupou o quinto lugar entre as localizações de câncer primário na mulher ${ }^{9}$.

Os estudos realizados por Creasman et al. ${ }^{6} \mathrm{e}$ pelo Grupo de Oncologia Ginecológica Americano ${ }^{7}$, demonstraram a importância de um estadiamento cirúrgico para o câncer de endométrio ${ }^{4}$. As taxas de metástases em linfonodos nas pacientes com tumores considerados iniciais são tanto mais elevadas, quanto maior a invasão miometrial e quanto maior o grau de diferenciação ${ }^{6,8,12}$. Segundo 
Childers et al. ${ }^{3}$ a metástase linfonodal é o fator prognóstico mais significante. Assim, a avaliação dos linfonodos regionais é importante para o planejamento terapêutico.

O tratamento tradicional do câncer de endométrio consiste na histerectomia total abdominal, salpingo-ooforectomia bilateral, citologia do líquido lavado peritoneal e linfadenectomia pélvica. A histerectomia vaginal com a remoção dos anexos é reservada a uma pequena fração de pacientes com esta doença, uma vez que a cirurgia vaginal não permite uma adequada exploração da cavidade peritoneal e nem acesso aos linfonodos pélvicos ${ }^{2}$.

Com o emprego da técnica descrita recentemente para linfadenectomia pélvica laparoscópica transperitoneal ${ }^{16,17}$, vimos estadiando pacientes com câncer de endométrio desde $1993^{1}$. Para estas pacientes, um completo estadiamento cirúrgico e histopatológico é obtido por uma combinação de cirurgia laparoscópica e vaginal.

O objetivos desta trabalho é de avaliar os resultados desta técnica quanto à acurácia para estadiamento e taxa de complicações.

\section{Pacientes e Métodos}

Durante o período de fevereiro de 1996 à fevereiro de 1998, doze pacientes com câncer de endométrio foram submetidas a linfadenectomia pélvica associada a histerectomia radical vaginal assistida por laparoscopia no Hospital de Ensino da Faculdade de Medicina do ABC e no Hospital Jaraguá. A média de idade das pacientes foi de $58,1 \pm 8,1$ (46 - 70) anos, o número médio de gestações foi $2,3 \pm 1,8(0-5)$ e o indice de massa corpórea médio $28,6 \pm 6,5(20,7-42,1)$.

O preparo intestinal, pré-operatório, consistiu da aplicação de dois enteroclismas um dia antes da cirurgia. Como profilaxia da trombose venosa profunda foi prescrito heparina subcutânea um dia antes da cirurgia, mantendo-a até um dia após a realização da mesma. Para profilaxia de infecções foram aplicadas uma dose de cefalotina (2 g intravenoso), no início do procedimento e após 4 horas.

Após a indução da anestésica, realizou-se a sondagem nasogástrica, a fim de eliminar todo o conteúdo gástrico. As pacientes foram então colocadas em posição de litotomia modificada, com ambos os joelhos no nivel da crista ilíaca, com as pernas abduzidas e rodadas, apoiadas nas perneiras. O suporte para o ombro foi colocado do lado esquerdo da paciente, sendo o braço homolateral apoiado ao lado do corpo e fixado por faixa crepe. Após rigorosa assepsia, realizou-se cateterismo vesical com Folley 14, que permaneceu por 24 horas.

A cavidade peritoneal foi adentrada por uma incisão infra-umbilical ${ }^{15}$. Procedeu-se a realização do pneumoperitônio com $\mathrm{CO}_{2}$ evitando-se que a pressão intra-abdominal não excedesse $14 \mathrm{mmHg}$. Após a inserção de um laparoscópio de $11 \mathrm{~mm}$ e observação do apêndice, da superficie do figado e dos órgãos genitais internos, foram feitas duas incisões de $10 \mathrm{~mm}$ a $3 \mathrm{~cm}$ medialmente de cada crista iliaca anterior pelas quais trocartes foram inseridos no abdome da paciente sob visão direta. Mais duas incisões adicionais de $5 \mathrm{~mm}$ foram feitas a meia distância da linha que une as punções umbilical e inguinal, de cada lado, lateralmente aos vasos epigástricos inferiores.

As pacientes foram colocadas em posição de Trendelenburg $\left(20^{\circ}-30^{\circ}\right)$. Antes de iniciar a linfadenectomia pélvica propriamente dita, procedeu-se a coleta de líquido peritoneal para exame citológico. A cirurgia iniciou-se com a cauterização das trompas, com o intuito de evitar a disseminação de células neoplásicas para cavidade pélvica.

Os procedimentos para a histerectomia com salpingo-ooforectomia bilateral consistiram da cauterização e secção dos ligamentos infundíbulopélvicos, redondos, artérias uterinas, ligamentos útero-sacros e liberação da reflexão vesical do útero. Pela via vaginal fez-se uma incisão circular na mucosa vaginal, em volta do colo, seguida de dissecção da fáscia pré-vesical e pré-retal. A retirada do útero foi feita com ligadura de paramétrios pela via vaginal. Não foi deixado dreno e a cúpula vaginal foi suturada por via vaginal.

Para realização da linfadenectomia deve-se ter em mente os limites de dissecção que são: anteriormente a veia circunflexa, posteriormente a bifurcação dos vasos iliacos, lateralmente os vasos iliacos externos, medialmente o ureter. O limite profundo de dissecção é a fossa obturadora, com o nervo obturador.

A linfadenectomia foi realizada de cada lado como descrito a seguir. Os vasos ilíacos e a artéria umbilical foram identificados sob a superficie do peritônio pélvico entre o ligamento redondo e o infundíbulo pélvico. O ligamento redondo foi apreendido, cauterizado e seccionado e o peritônio foi aberto de maneira que toda a área entre os ligamentos redondo e infundíbulo pélvico seja exposta. O espaço para vesical é aberto e então ampliado por dissecção romba entre a artéria umbilical medialmente e os vasos ilíacos externos lateralmente. $\mathrm{O}$ assoalho pélvico é desta forma facilmente alcançado sem nenhum sangramento. $\mathrm{O}$ instrumento que apreendia o ligamento redondo 
é então liberado, fechado, e colocado no espaço paravesical, de modo a retrair a artéria umbilical medialmente. Os tecidos com linfáticos abaixo da veia ilíaca externa é então claramente visivel e pode ser seguramente dissecada. O nervo obturador é identificado e dissecado caudalmente até o ponto em que deixa a pelve. O liberação da porção inferior da veia ilíaca externa é realizada por dissecção romba. O tecido linfático, entre o nervo obturador e a veia ilíaca externa, é então apreendido e completamente separado da parede pélvica. Neste ponto, o músculo obturador interno é exposto. Deve se evitar lesões das veias retropúbicas e as anastomoses venosas entre as veias obturadora e ilíaca externa. A magnificação que é obtida pelo laparoscópio, colocado a uma distância próxima ao local cirúrgico, permite dissecção precisa destes vasos. A porção caudal do pedículo do tecido conjuntivo é então liberado da área do forame obturador e do canal femoral através de tração suave. A borda do tecido é firmemente apreendida e movida cranialmente, sendo cuidadosamente dissecada da veia e artéria ilíaca externa lateralmente e da artéria umbilical, medialmente. Neste ponto, a borda linfática esta somente aderida a área de bifurcação interilíaca, sendo gentilmente separado por tração e dissecção romba. A amostra do tecido linfático é então removida do abdome pelo trocarte de $10 \mathrm{~mm}$. A mesma técnica é realizada de outro lado. A hemostasia é então checada. No final do procedimento, o peritônio é deixado aberto para permitir a drenagem de fluído linfático para dentro do abdome.

Nos casos em que havia invasão de colo, o ureter foi isolado do paramétrio por via laparoscópica e a parametrectomia feita por via vaginal utilizando técnica semelhante à descrita por Schauta.

Nos casos em que a biópsia prévia demonstrava um tumor GI e a invasão miometrial foi constatada após a retirada da peça, a linfadenectomia era feita após a sutura da cúpula vaginal quando o pneumoperitônio era refeito.

\section{Resultados}

Dos doze casos operados, nove foram estadiados como Ib, um como IIa, um como IIb e um como IIIa, conforme critérios recomendados pela FIGO.

Uma paciente evoluiu com aumento da pressão endotraqueal, sendo necessário abandonar o procedimento, e realizar a linfadenectomia laparoscópica seguida de laparotomia para o término da cirurgia. Não foram observadas complicações intra-operatórias relacionadas ao ato cirúrgico. Das onze pacientes submetidas a linfadenectomia pélvica associada a histerectomia radical vaginal assistida por laparoscopia, apenas uma evoluiu com granuloma de cúpula vaginal no pós-operatório.

O tempo anestésico médio foi de 4,8 $\pm 1,4$ horas (com variação de 3 a 8 horas). Este tempo incluiu o tipo de procedimento: linfadenectomia pélvica mais histerectomia radical vaginal assistida por laparoscopia. O tempo médio de internação foi de 3,3 $( \pm 1,3)$ dias (com variação entre 2 e 5 dias).

O número total de linfonodos retirados foi de 176, sendo $104(59,1 \%)$ do lado direito e 72 (40,9\%) do lado esquerdo. Não foram encontrados linfonodos comprometidos. O número médio de linfonodos retirados por paciente foi de 18,5 $\pm 9,5$ $(8-40)$, sendo a média do lado direito de $10,1 \pm$ $5,8(4-25)$ e a do lado esquerdo de 8,4 \pm 4 (4 - 17). Estes resultados estão apresentados na Tabela 1.

\section{Discussão}

Embora existam numerosos fatores prognósticos para pacientes com câncer de endométrio, a presença de metástase é a mais importante ${ }^{3}$. A presença de metástase para linfonodos pélvicos não é somente um sinal de mau prognóstico, mas também implica em mudanças no tratamento destas pacientes. Por isto, uma avaliação precisa dos linfonodos regionais é importante para o planejamento terapêutico.

As pesquisas por técnicas mais sensiveis e específicas têm conduzido os estudiosos a avaliar os métodos radiológicos para detecção de metástase linfonodal ${ }^{3}$. Infelizmente, a efetividade da maioria dos modernos métodos radiológicos é inferior à da cirurgia de estadiamento.

Segundo Kindermann ${ }^{11}$, somente se observa uma alta taxa de envolvimento linfonodal de até $30 \%$, quando ocorre invasão profunda do miométrio. Mas mesmo neste grupo desfavorável, a maioria das pacientes $(70 \%)$ têm linfonodos pélvicos livres, não necessitando radioterapia pélvica. Embora o número de pacientes por nós operadas seja pequeno, não se constatou metástase linfonodal em nossa casuística.

O estadiamento cirúrgico-patológico tornouse mais indicado para as doenças malignas por causa da imprecisão do estadiamento clínico. Demonstrou-se que quando comparamos com o estadiamento cirúrgico, uma significativa porcentagem de pacientes com doença maligna na pelve são subestadiadas clinicamente ${ }^{3}$. Estas discrepância pode chegar a $40 \%{ }^{18}$. 
Tabela 1 - Características da pacientes, cirurgia executada e número de gânglios dissecados à Direita e a Esquerda.

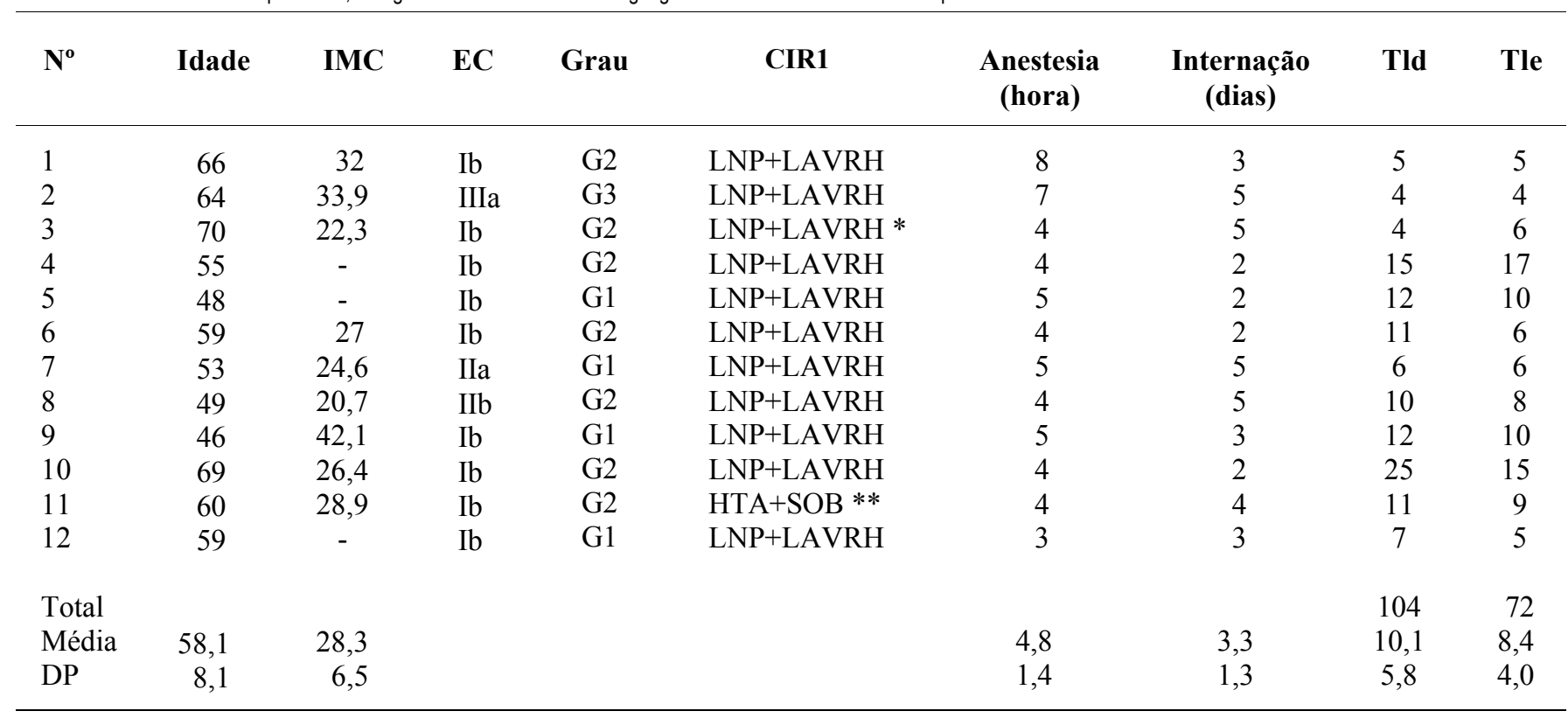

* Pós-op: Granuloma de cúpula vaginal

** Planejado LNP+LAVRH: evoluiu com aumento da pressão endotraqueal

IMC = Índice de Massa Corpórea

LNP = Linfadenectomia Pélvica laparoscópica

LAVRH = Histerectomia Radical Vaginal Assistida por Laparoscopia

TId = Número Total de Linfonodos Direito Ressecados

Tle = Número Total de Linfonodos Esquerdo Ressecados

Em relação ao status dos linfonodos, o estadiamento cirúrgico pode acrescentar informações que podem influenciar o prognóstico e tratamento. A presença de citologia peritoneal positiva ou de metástases anexiais ou intraperitoneais alteram a conduta. A ocorrência de doenças inflamatórias como a diverticulite ou infecções anexiais presentes ou passadas, assim como as aderências pélvicas, podem influenciar no resultado da radioterapia. Todas estas informações podem ser obtidas laparoscopicamente. Além disso as complicações com este procedimento, inicialmente parecem ser aceitáveis ${ }^{2,3}$.

Herd et al. ${ }^{10}$ apresentaram resultado de seis pacientes tratadas laparoscopicamente com dissecção de oito linfonodos pélvicos. Childers et al. ${ }^{4}$ obtiveram uma média de 17,5 linfonodos em 13 pacientes. Já Massi et al. ${ }^{14}$ apresentaram uma série com 106 pacientes que foram submetidas a linfadenectomia por via clássica, tendo obtido uma média de 22 linfonodos. Como podemos observar, a linfadenectomia laparoscópica, tem resultados semelhantes aos da via abdominal. Isto é claro em nossa amostragem uma vez que obtivemos uma média de 18,5 linfonodos por paciente.

Em relação ao comprometimento linfonodal, Massi et al. ${ }^{14}$ e Lin et al. ${ }^{13}$ relataram a presença de
4\% de linfonodos pélvicos comprometidos em pacientes com adenocarcinoma de endométrio tratadas por via abdominal. Childers et al. ${ }^{4}$ e Herd et al. ${ }^{10}$ apresentam comprometimento de $3 \%$ dos linfonodos, para pacientes tratadas por laparoscopia. Em nossa casuística, nenhuma paciente apresentou comprometimento linfonodal.

Childers et al. ${ }^{3}$ referem que $98 \%$ dos linfonodos podem ser obtidos laparoscopicamente e que o tempo para realizar uma linfadenectomia pélvica e paraaórtica é de 2 horas ou menos, com sangramento mínimo. Massi et al. ${ }^{14}$ observaram que suas pacientes tratadas cirurgicamente pela via abdominal ficaram internadas por 5,5 dias em média. O nosso tempo médio de internação foi de 3,3 dias. A literatura mostra que o tempo de internação hospitalar é menor para as pacientes que são submetidas a linfadenectomia ${ }^{2,3}$. Além disto, mais importante do que o tempo de internação é o tempo de recuperação mais rápido que acompanha a cirurgia laparoscópica.

É possível o início precoce da radioterapia para as pacientes tratadas laparoscopicamente em oposição àquelas que são submetidas a uma laparotomia.

O sucesso do estadiamento laparoscópico depende de múltiplos fatores. Existe vantagem se 
este procedimento for realizado em uma instituição onde as pessoas que trabalham na sala cirúrgica estejam familiarizadas com este procedimento. Além disto, é importante que o cirurgião seja um laparoscopista e oncologista bem treinado e que o equipamento cirúrgico seja adequado. Além disso, a seleção e o preparo das pacientes são extremamente importantes. A obesidade, o inadequado preparo intestinal e as aderências intraperitoneais são fatores que tornam a linfadenectomia mais dificil. No entanto, em nossa casuística, esses fatores não impediram a sua realização. As pacientes e os médicos, no entanto, devem ser lembrados de que esta técnica ainda se encontra sob investigação.

\section{SUMMARY}

Purpose: to demonstrate a new approach to treatment of endometrial cancer.

Methods: Between February, 1996 and February, 1998, twelve patients with endometrial cancer, diagnosed by hysteroscopy and biopsy, were submitted to pelvic lymphadenectomy and hysterectomy with salpingo-oophorectomy by laparoscopy. The mean age was 58.1 years, the mean number of gestations was 2.3 and the mean body mass index was 28.6.

Results: the mean length of anesthesia was 4.8 hours. The mean time of hospital stay was 3.3 days. The total of lymph nodes obtained was 176, 104 (59.1\%) being from the right side and 72 (40.9\%) from the left side. The mean of lymph nodes per patient was 18.5. We observed two complications: in one case the laparoscopic procedure had to be abandoned because the patient presented a dangerous increase in intratracheal pressure and in the other case a granuloma in the vagina was observed.

Conclusions: the initial evaluation of the laparoscopic hysterectomy and laparoscopy shows that we achieved good results regarding the accuracy of staging, the number of nodes and a small number (3) of complications.

KEY WORDS: Laparoscopy. Hysterectomy. Lymphadenectomy. Surgery: complications. Endometrium: carcinoma.

\section{Referências}

1. Barbosa CP, Ruano JMC, Napolitano AC. Linfadenectomia laparoscópica e histerectomia vaginal assistida por laparoscopia no tratamento do câncer de Endométrio. RBM - Ginecologia e Obstetrícia 1995; 6:187-90.

2. Childers JM, Surwit EA. Combined laparoscopic and vaginal surgery for the management of two cases of stage I endometrial cancer: A case report Ginecol Oncol 1992; 45:46-51.
3. Childers JM, Hatch KD, Surwit EA. The role of laparoscopic lymphadenectomy in the management of cervical carcinoma. Gynecol Oncol $1992 ; 47: 38-43$.

4. Childers JM, Spirtos, NM, Brainard P, Surwit EA. Laparoscopic staging of the patient with incompletely staged early adenocarcinoma of the endometrium. Obstet Gynecol 1994; 83:597-600.

5. Creasman WT. New gynecologic cancer staging. Obstet Gynecol 1990; 75:287-8.

6. Creasman WT, Boronow RC, DiSaia PJ, Blessing J. Adenocarcinoma of the endometrium: Its metastatic lymph node potential. Gynecol Oncol 1976, 4:239-43.

7. Creasman WT, Morrow CP, Bundy BN, Homesley HD, Graham JE, Heller PB. Surgical pathological spread pattern of endometrial carcinoma: A Gynecologic Oncology Group study. Cancer 1987; 60:2035-41.

8. DiSaia PI, Creasman WT (eds.). Adenocarcinoma of the uterus. In: Clinical Gynecologic Oncology. New York: Churchill Livingstone 1995.

9. Franco ELF, Alves EC, Saltz E, Abreu E, Pinheiro LF, Rezende MCR et al.. Registro Nacional de Patologia Tumoral - Diagnóstico de Câncer no Brasil - 19811985. São Paulo: BSB Editores, $1^{\text {a }}$ ed., 1991. p.324.

10.Herd J, Fowler JM, Shenson D, Lacy S, Montz FJ. Laparoscopic para-aortic lymphonode sampling: Development of a technique. Gynecol Oncol 1992; 44:271-6.

11.Kindermman, G. Endometrial cancer. Surgical treatment and results In: Burghardt, Managhan, Kindermman, Web, Surgical Gynecological Oncology. Stuttgart, New York: Thieme. 1993. p.281.

12.Lewis B, Stallworthy JA, Cowdell B. Adenocarcinoma of the body of the uterus. J Obstet Gynaec Br Comm 1970; 77:343-6.

13.Lin HH, Chen CD, Chen CK, Chen CL, Chow SN, Huang SC. Is total abdominal hysterectomy with bilateral salpingo-oophorectomy adequate for new FIGO stage I endometrial carcinoma? Br J Obstet Gynaecol 1995; 102:148-52.

14.Massi G, Savino L, Susini T. Vaginal hysterectomy versus abdominal hysterectomy for the treatment of stage I endometrial adenocarcinoma. Am J Obstet Gynecol 1996; 174:1320-6.

15.Palmer, R. Safety in Laparoscopy. J Reprod Med $1974 ; 13: 1-5$

16.Querleu D, LeBlanc E, Castellain B. Laparoscopic pelvic lymphadenectomy in the staging of early carcinoma of the cervix, Am J Obstet Gynecol $1991 ; 164: 579-81$

17.Reich H, McGlynn F, Wilkie W. Laparoscopic management of stage I ovarian cancer: A case report, J Reprod Med 1990; 35:601-4.

18. Recio FO, Piver S Hemplling RE. Pretreatment transperitoneal laparoscopic staging pelvic and paraaortic lymphadenectomy in large $(\geq 5 \mathrm{~cm})$, Stage Ib2 cervical carcinoma: Report of a pilot study. Gynecol Oncol 1996; 63:333-6. 